\title{
Hot Times in the Hertzsprung Gap
}

\author{
THOMAS R. AYRES
}

Center for Astrophysics and Space Astronomy, Campus Box 389, University of Colorado, Boulder, CO 80309, USA

Moderate-mass giants represent a touchstone for probing the mechanisms of magnetic activity among fast-rotating convective stars. Extreme ultraviolet and soft X-ray observations of such stars detect generally hot coronae: the Hertzsprung-gap giants (F5-G2), in particular, have remarkable high-excitation peaks $\left(10^{7} \mathrm{~K}\right.$, or hotter) in their emission-measure distributions. While the high-temperature coronal plasmas are reminiscent of violent solar flares, the highenergy spectra of the Hertzsprung-gap giants appear to be quite steady over time; in contrast to other hot-corona objects whose optical light curves carry the stamps of starspots, and whose high-excitation emissions are sporadically-and dramatically-variable. The constancy of the Hertzsprung-gap stars is particularly puzzling in light of high-dispersion FUV spectroscopy that reveals supersonic flows in their $10^{5} \mathrm{~K}$ subcoronal "transition zones."

\section{Introduction}

The exploration of high-excitation coronae among Main Sequence late-type stars is relatively advanced; not surprising given that we have a prime example-the Sun-close at hand (e.g., Cox, Livingston \& Matthews 1991). As one moves away from the MS, however, the situation becomes more complex; in part because of the dramatic structural changes imposed by the rapid post-MS evolution of moderate-mass $\left(\approx 2-4 M_{\odot}\right)$ stars. One key group are the G8-K0 giants in the "Clump" at the base of the red giant branch. Most of such stars are in the post-flash core-helium burning phase. A second key group are the F5-G2 giants in the Hertzsprung gap, blueward of the Clump. They are the predecessors of the Clump stars, and are relatively rare thanks to the brief first-crossing time for moderate-mass giants. The denizens of the gap are the fresh descendants of Aand B-type dwarfs; consequently one finds many fast-rotators among them. Because the gap giants also are convective, one might anticipate enhanced spin-catalyzed magnetic activity. However, just redward of G0 is a sharp break in the rotational speeds of the giants (Gray 1989). The braking mechanism is controversial. Internal redistribution of angular momentum certainly plays a role, but magnetospheric mass loss (crucial for dwarf stars) might be important as well (e.g., Schrijver \& Pols 1993: hereafter SP).

A pivotal aspect of the Hertzsprung-gap stars, originally noted by Simon and Drake (1989: hereafter SD), is that they appear to be deficient in their X-ray luminosities compared with the coronal proxy C IV $\lambda 1549$. At the same time, the active Clump giants are more solar-like in their X-ray/C IV ratios. Exploring the dichotomy with the powerful tools provided by the EUVE and $H S T /$ GHRS has provided new clues to the nature of the underlying magnetic "engine" that powers stellar activity.

\section{ROSAT and IUE Observations of Coronal Giants}

Although the first recognition of the abnormal coronae of the Hertzsprung-gap giants came from Einstein pointings, the recent ROSAT all-sky survey has provided a clear, and minimally-biased, view of the dichotomy. Figure 1 illustrates an X-ray H-R diagram based on material from the "RIASS" campaign (which coordinated UV spectroscopy by the IUE satellite with the ROSAT survey passes: see Ayres et al. 1995).

Figure 2 compares the normalized fluxes $\left(f / f_{\text {bol }}\right)$ of X-rays and C IV $\lambda 1549$ for the 


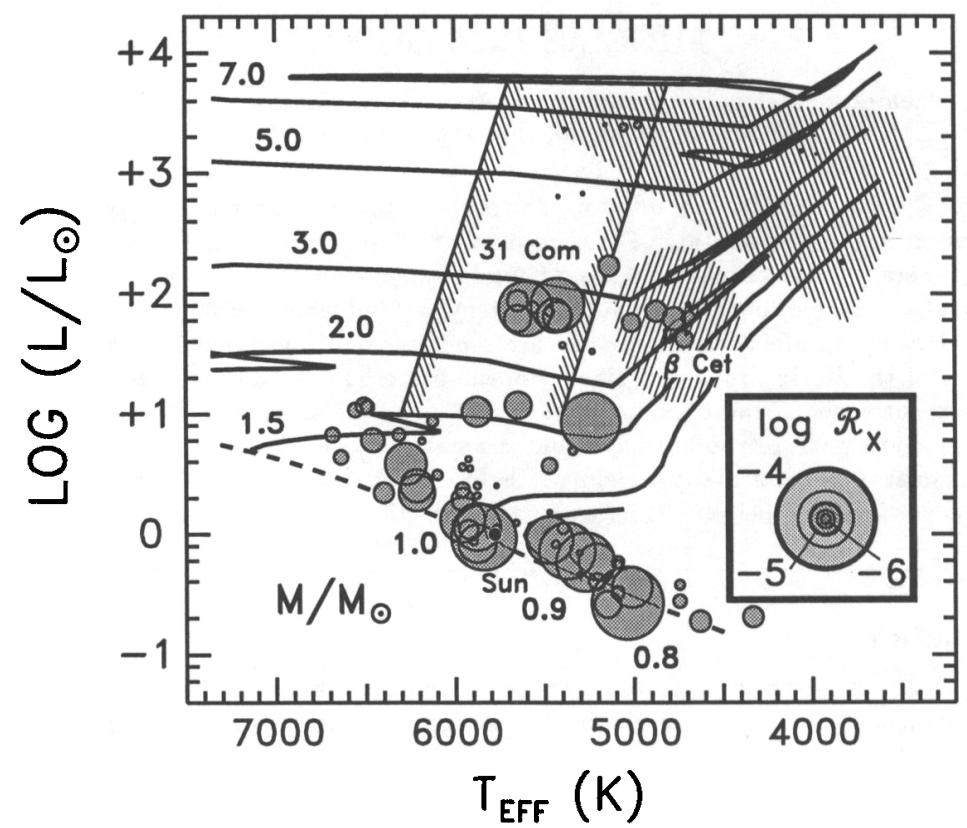

FIGURE 1. X-ray H-R diagram. Size of each "bubble" is proportional to $\mathcal{R}_{\mathrm{X}} \equiv f_{\mathrm{X}} / f_{\text {bol }}$ according to key. Slanted hatched lines delimit the Hertzsprung gap; hatched oval, the "Clump;" shaded wedge, the "hybrid-chromosphere" stars (not discussed here). Solid curves depict evolutionary trajectories; dashed curve is ZAMS. Dot at base of $1 M_{\odot}$ track indicates the Sun.

evolved stars of the RIASS sample. The thick dashed line depicts the power-law correlation obeyed by dwarfs of solar-type and cooler. The Clump giants generally follow the MS trend, but the Hertzsprung-gap stars (as well as supergiants of all types) fall below it. The behavior is ascribed to an X-ray deficiency, because $\mathrm{C} \mathrm{IV/Mg} \mathrm{II} \mathrm{diagrams} \mathrm{are}$ perfectly normal for the F5-G2 giants (and G/K supergiants: Ayres et al. 1995).

SD offered an explanation for the X-ray deficiency of the warm giants based on classical acoustic-wave heating of their coronae, rather than the magnetic agency believed to operate in solar-type stars and active late-type binaries. The latter all show evidence for starspots, flares, and other signatures of solar-like magnetic phenomena. In the SD model, the acoustic waves strongly heat the intermediate temperature layers (up to, say, a few $\times 10^{5} \mathrm{~K}$ ), but the lack of closed magnetic loops permits the heated gas to flow away from the star in a pervasive but tenuous wind. The low densities of the expanding exospheric flow depress the emission measure $\left(E M \equiv \int_{V} n_{\mathrm{e}}^{2} d V\right.$ ) relative to a normal magnetically-bottled (closed-field) corona, yielding a much reduced $\mathrm{X}$-ray luminosity. At the same time, the wind-particularly if slightly magnetized--sheds considerable angular momentum from the initially fast-rotating star, promoting the dramatic spindown seen at the "GO break." The acoustic heating model also explains the apparent constancy of the FUV emissions of the Hertzsprung-gap giants (e.g., Ayres et al. 1995), noted originally in the Capella system ( $\alpha$ Aur = HD 34029: G8 III + G1 III; see, e.g., Ayres (1988), and references therein). Because the acoustic heating is a byproduct of the global convective 


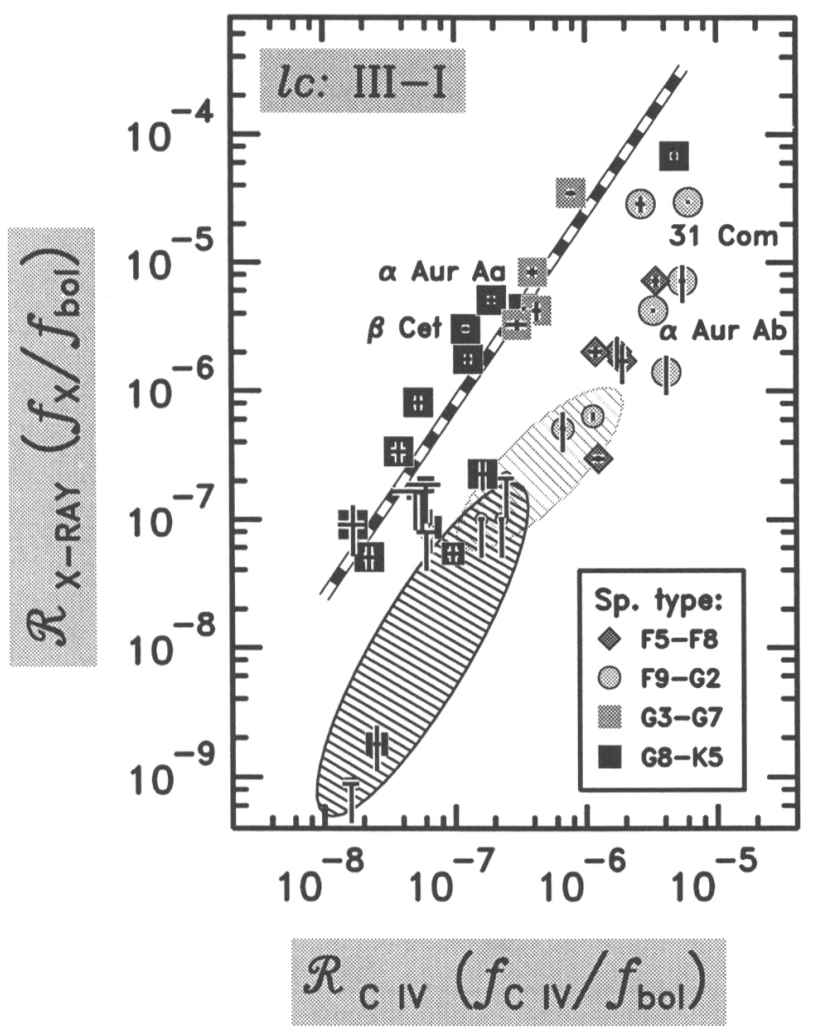

FiguRE 2. X-ray/C IV "correlogram" from RIASS campaign. Luminosity classes III-I are depicted explicitly; thick dashed line is mean power-law trend for F9-K5 dwarfs. Larger symbols indicate class III; intermediate-size symbols, II-I; small symbols with downward "tails," upper limits. Middle (light) oval encompasses the G supergiants; lower (dark) oval, the K-type hybrids. Symbol for G8 primary of Capella ( $\alpha$ Aur Aa) lies on dashed curve, partially obscured.

envelope, it should be comparably uniform in its surface effects. In contrast, solar magnetic fields erupt in localized active regions that ultimately can produce hemispherical asymmetries in $\mathrm{X}$-ray or UV irradiances.

\section{New Insights from the Extreme Ultraviolet}

A recent breakthrough in the exploration of the X-ray deficiency "syndrome" came with the $E U V E$ satellite (Bowyer \& Malina 1991). The key binary Capella was a calibration target for the spectroscopy modes, and an early $80 \mathrm{ks}$ pointing was reported by Dupree et al. (1993). The Capella SW and LW spectra are dominated by hot iron lines, with an apparent strong peak in the $E M$ distribution at $6 \times 10^{6} \mathrm{~K}$. Unfortunately, however, the Capella EUV spectra are an unknown blend of the coronal emissions of the two companions (which might be more nearly equal in the EUV: the large FUV advantage of the G1 secondary might be offset by its X-ray deficiency, e.g., Ayres 1988). Nevertheless, 


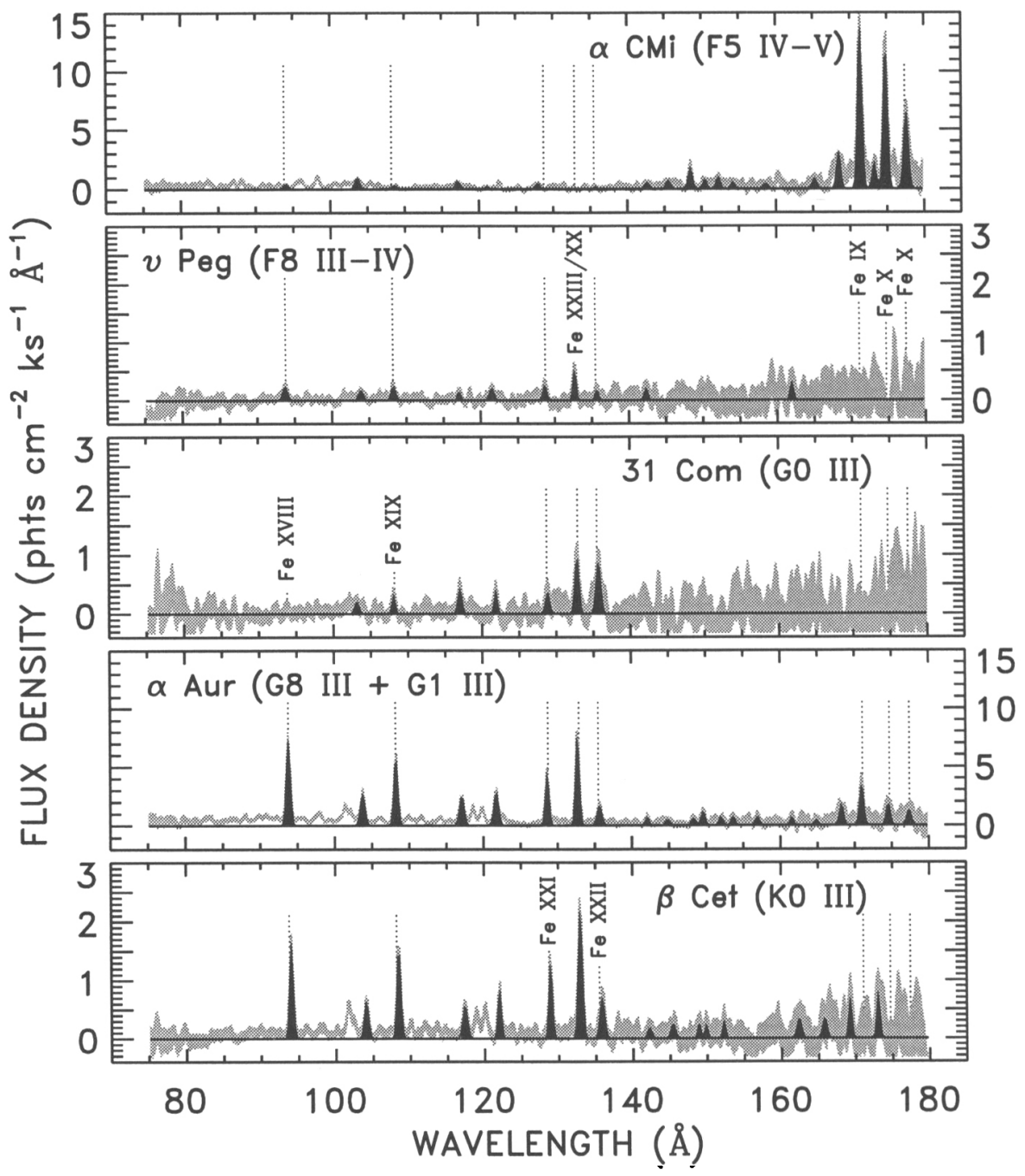

FIGURE 3. EUVE SW spectra of Hertzsprung-gap and Clump giants. Light shaded envelopes are traced spectra $\pm 1 \sigma$; solid peaks represent detections of selected "features." The instrumental sensitivity has been corrected, but there was no compensation for interstellar absorption.

the Capella spectra show no evidence for the very soft $\left(T<10^{6} \mathrm{~K}\right)$ coronal component predicted by the acoustic-heating scenario (cf., SD).

Another of the EUVE targets during the early Guest Observer phase was 31 Comae (HD 111812: G0 III), an archetype gap star. Remarkably, its emissions (in an $80 \mathrm{ks}$ pointing) are completely dominated by hyper-ionized iron, with little material cooler than about $10^{7} \mathrm{~K}$. Figure 3 compares the EUVE SW spectra of Capella and $31 \mathrm{Com}$ with those of Procyon (F5 IV-V; a cool-corona dwarf like the Sun), $\beta$ Ceti (K0 III; a Clump 

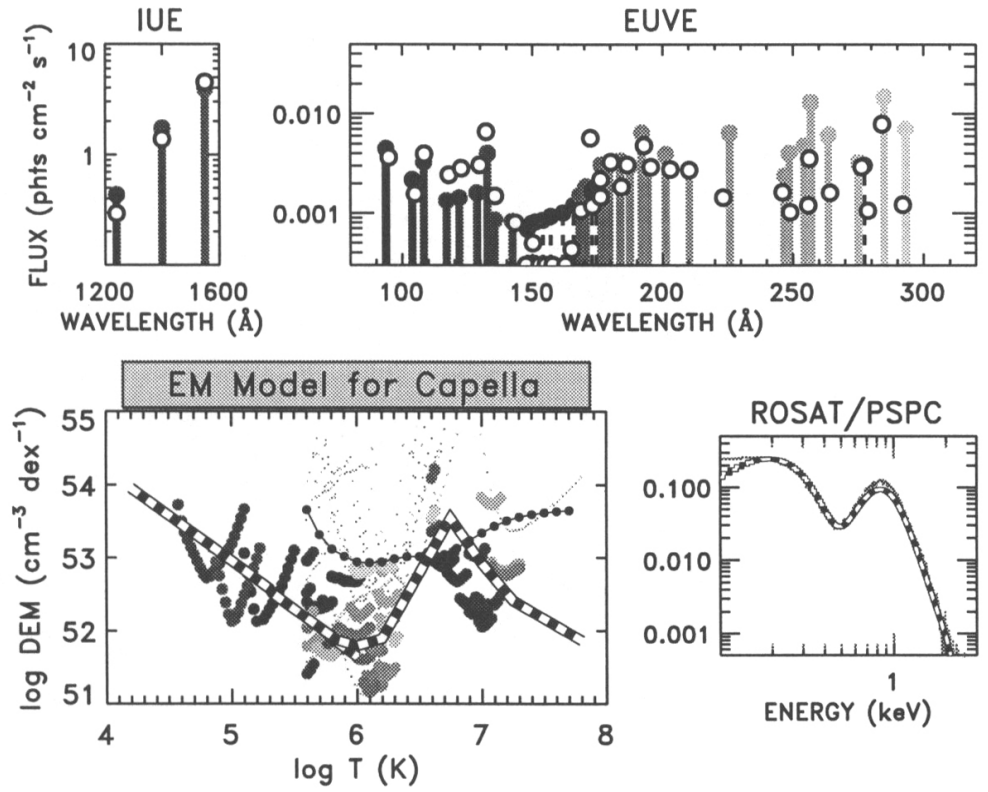

Figure 4. Emission-measure modeling of Capella based on EUVE SW and MW spectra, constrained by IUE and ROSAT fluxes. In the upper panels, FUV and EUV detections (corrected for ISM absorption) are marked by vertical solid bars; upper limits by dashed bars. ROSAT PSPC pulse-height spectrum in lower right panel is a light-shaded band (barely visible here): observed counts (per $0.010 \mathrm{keV}$ bin) $\pm 1 \sigma$. Open circles in IUE and $E U V E$ frames, and dashed curve in ROSAT frame, are predictions based on EM model in lower left panel. In it, heavy dashed curve is model; larger dots are "monothermal" curves for individual spectral features (connected dots for PSPC); small dots depict upper limit curves for nondetections.

giant like the G8 primary of Capella), and $v$ Pegasi (F8 III-IV; another Hertzsprung-gap giant, observed recently in AO-2). It is clear that while the Hertzsprung-gap stars might be $\mathrm{X}$-ray deficient, they are no slouches coronal-temperature-wise.

\section{Emission-Measure Modeling}

Figures 4 and 5 illustrate the inferred $E M$ distributions for Capella and 31 Comae, respectively, based on fitting $I U E$ fluxes of key FUV species at the low-temperature end; $E U V E$ lines at the high-temperature end; and ROSAT PSPC pulse-height spectra as a secondary constraint. The EUV modeling was based on prominent "features" (e.g., Fig. 3); that is, a specific set of wavelengths where there appears, over one or more temperature intervals, a strong emission line (not necessarily from the same species over the full temperature range). Emissivity curves were developed for each "feature" by a width-constrained measurement of temperature-resolved spectra simulated with the line list of Mewe, Gronenschild \& van den Oord (1985), assuming cosmic abundances. The philosophy differs somewhat from the approach of assigning a specific identification (and thus emitting species) to an apparent detected peak in the EUVE spectra, but 

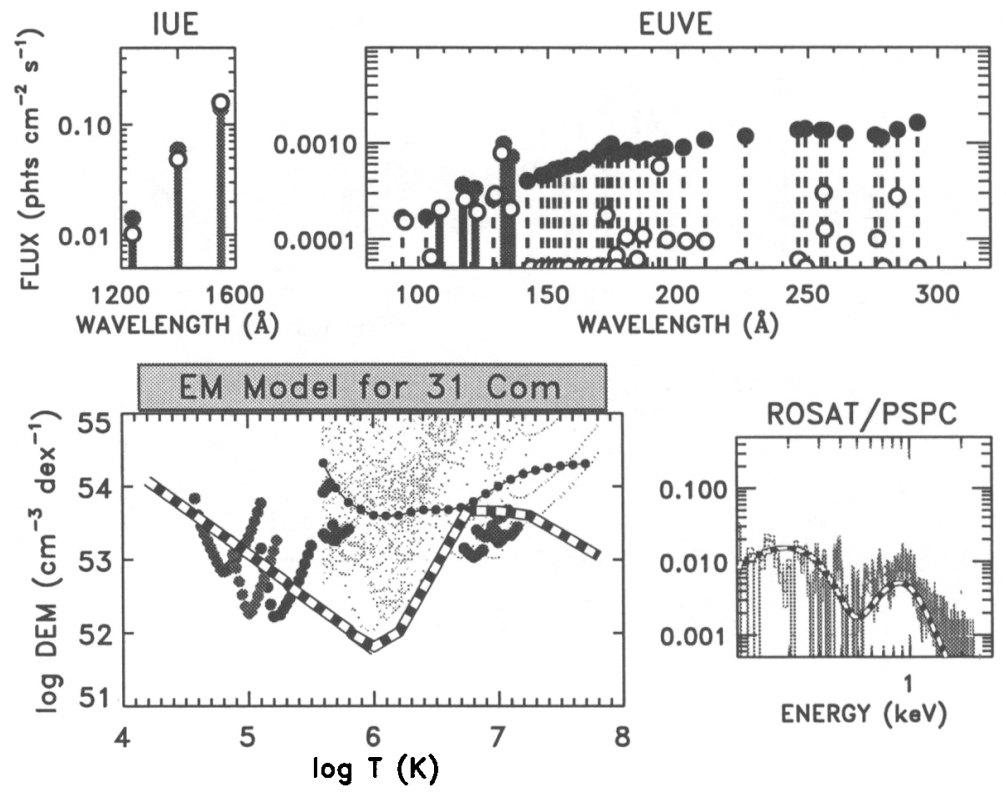

FIGURE 5. Same as previous figure, for 31 Comae. PSPC observation was taken with Boron filter, whose response has been corrected.

neatly avoids the quandary of blended lines and/or ambiguous assignments. The iterative $\chi^{2}$ minimization procedure-with a weighting scheme to accommodate upper limitsempirically describes the coronal $E M$ distribution in sufficient detail to compare with theoretical expectations, given the uncertainties in the underlying atomic physics (cf., discussions at this meeting), and the further ambiguities posed by possible abundance anomalies (cf., the "FIP" effect) and gas dynamics.

\section{Discussion and Conclusions}

On the one hand, the very hot coronae of the Hertzsprung-gap giants argues forcefully against the acoustic heating scenario. $1 \mathrm{keV}$ gas implies very large post-shock velocity contrasts, thousands of $\mathrm{km} \mathrm{s}^{-1}$. But, as illustrated in figure 6, the attendant line Doppler broadening is not seen in high-dispersion HST/GHRS spectra. On the other hand, the presence of solar-flare temperature material and supersonic flows, but without the overt signatures of flare activity, presents a dilemma for the magnetic heating scenario.

One way out is to appeal to the nanoflare hypothesis (e.g., Parker 1988). Namely, the magnetosphere of a typical Hertzsprung-gap giant is in a continual state of flaring, but the demographics of the flares is dominated by numerous, relatively compact, widely dispersed events. Such a population would be equivalent to that proposed for heating the solar corona (Parker 1988), but without the tail towards larger events evident in the Sun's case. The truncated population might be a natural consequence of the fast rotation 


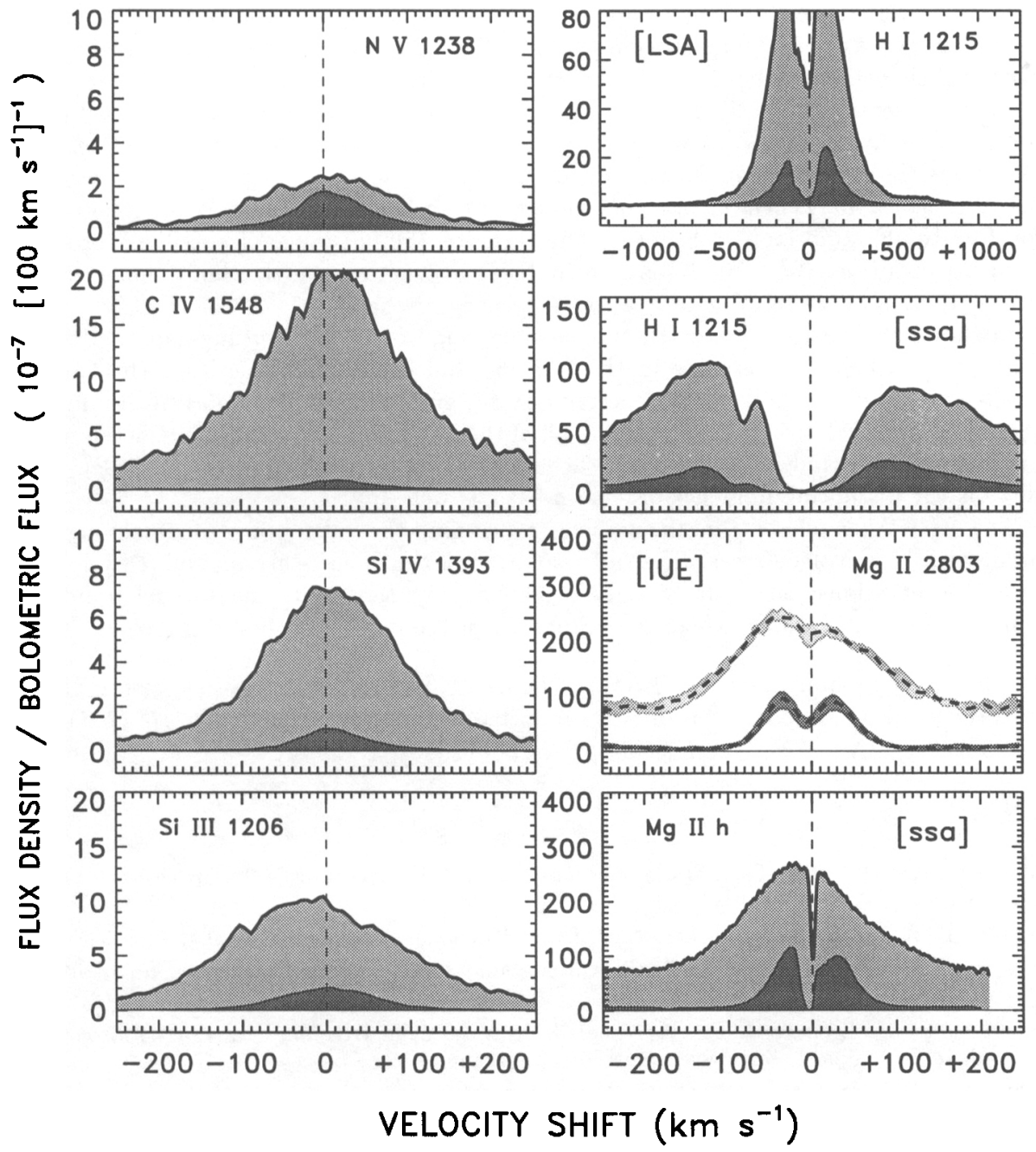

FIgure 6. High-dispersion UV spectra of 31 Com (light shaded) and $\beta$ Cet (dark shaded). Data are taken from $H S T / G H R S$, except for Mg II $\lambda 2803$ panel labeled "IUE." There, shaded envelopes represent $\pm 1 \sigma$ (temporal) deviations of available $\mathrm{Mg}$ II profiles over 15-year span of IUE mission. In upper left panels, the anomalous strength of $\mathrm{N} \mathrm{V}$, and weakness of $\mathrm{C} I \mathrm{~V}$, in $\beta$ Cet probably are due to "evolved" abundances. Note redshifts of N V, C IV, and Si IV in both stars, but apparent blueshifts of $\mathrm{Mg}$ II $h$ and H I Ly $\alpha$ in 31 Com. Despite partial blurring of 31 Com lineshapes by fast rotation $\left(v \sin i \approx 70 \mathrm{~km} \mathrm{~s}^{-1}\right)$, the large Doppler widths indicate supersonic motions in $T \approx 10^{5} \mathrm{~K}$ regime. The profiles show no evidence, however, for 1000's of $\mathrm{km} \mathrm{s}^{-1}$ broadening expected for acoustic waves of sufficient amplitude to produce $1 \mathrm{keV}$ coronal material detected by $E U V E$. 
of the Hertzsprung-gap giants coupled with their thin convective envelopes: a limiting variant of Dynamo action.

If the nanoflare-ona mechanism applies to the Hertzsprung-gap giants, what about the Clump stars? After all, they too show a high- $T$ peak in their coronal $E M$ distribution, even if their overall multi-spectral properties (e.g., $\mathcal{R}_{\mathrm{X}} / \mathcal{R}_{\text {CIV }}$ ) are more solar-like. The author was reminded gently at the meeting that it is obvious that giant stars must have hot coronae, owing to their low surface gravities. Yet, most modern theories of coronal structures (i.e., constant pressure loops) dismiss the influence of surface gravity precisely because the plasma is field-dominated (see, e.g., Antiochos 1994; gravity is important only for $T \approx 10^{5} \mathrm{~K}$ "cool loop" solutions). Furthermore, the full range of possible "coronal" temperatures (i.e., $10^{5}-10^{7} \mathrm{~K}$ ) is seen in discrete structures on the Sun. Thus gravity, per se, must play a subordinate role to the heating mechanism.

In short, the puzzle of the structure and heating of the Hertzsprung-gap coronae is mirrored by their counterparts in the Clump. Indeed, it is curious that the Clump giants exhibit any high-excitation activity at all, given the ample opportunities during their post-MS evolution to lose virtually all of their Dynamo-catalyzing spin. Even weak magnetospheric winds in giant stars shed angular momentum prodigiously (SP), and there is the disrupting influence of helium flash as well.

The EUVE has offered tantalizing clues concerning the nature of coronae among the moderate-mass evolved stars. The full resolution of the open issues undoubtedly lies in future observations; particularly high-dispersion, high-sensitivity measurements in the 100-400 $\AA$ spectral range, where $E U V E$ has taken the pioneering first steps.

I thank my collaborators-A. Brown, S. Drake, T. Simon, and R. Stern-for their help with the project. My participation was supported by NASA grants NAG5-2274 (EUVE), GO-5323.01-93A (HST), and NAG5-2451 (ROSAT).

\section{REFERENCES}

ANTiochos, S. K. 1994, The physics of coronal closed-field structures, Adv. Space Res., 14(4), 139

AYres, T. R. 1988, A spectral dissection of the ultraviolet emissions of Capella, ApJ, 331, 467

AYRES, T. R., ET AL. 1995, The RIASS Coronathon: Joint X-ray and ultraviolet observations of normal F-K stars, ApJS, 96, 223

Bowyer, S. \& Malina, R. F. 1991, The EUVE Mission, in Extreme Ultraviolet Astronomy, ed. R. F. Malina \& S. Bowyer, New York: Pergamon, 397

Cox, A. N., Livingston, W. C. \& Matthews, M. S. (editors) 1991, Solar Interior and Atmosphere, Univ. of Arizona Press

Dupree, A. K., Brickhouse, N. S., Doschek, G. A., Green, J. C., \& Raymond, J. C. 1993, The extreme ultraviolet spectrum of Alpha Aurigae Capella, ApJL, 418, L41

GrAY, D. F. 1989, The rotational break for G giants, ApJ, 347, 1021

Mewe, R., Gronenschind, E. H. B. M. \& VAN DEN OORD, G. H. J. 1985, Calculated $\mathrm{X}$-radiation from optically-thin plasmas. V., A\&AS, 62, 197

PARKer, E. N. 1988, Nanoflares and the solar X-ray corona, ApJ, 330, 474

SchriJver, C. J. \& Pols, O. R. 1993, Rotation, magnetic braking, and dynamos in cool giants and subgiants, A\&A, 278, 51

Simon, T. \& Drake, S. A. 1989, The evolution of chromospheric activity of cool giants and subgiant stars, ApJ, 346, 303 